CARPATHIAN J. MATH.

Volume 37 (2021), No. 1,

Pages 127 - 133

\title{
Universal centers and composition conditions on the complex plane
}

\author{
Claudia Valls
}

ABSTRACT. We characterize the universal centers of the ordinary differential equations in the complex plane $d \rho / d \theta=\sum_{i=1}^{\infty} a_{i}(\theta) \rho^{i+1}$, where $a_{i}(\theta)$ are trigonometric polynomials with complex coefficients, in terms of the composition conditions.

\section{INTRODUCTION AND STATEMENT OF THE MAIN RESULTS}

We consider the differential equation

$$
\frac{d \rho}{d \theta}=\sum_{i=1}^{\infty} a_{i}(\theta) \rho^{i+1}
$$

on $(\rho, \theta) \in \mathbb{C} \times \mathbb{S}^{1}$ in a neighborhood of $\rho=0$ and where $a_{i}(\theta)$ are trigonometric polynomials in $\theta$ with complex coefficients.

Following the definitions in $\mathbb{R}$, we say that equation (1.1) determines a center if $\rho(0)=$ $\rho(2 \pi)$. The center problem consists on finding conditions on the coefficients $a_{i}$ under which this equation has a center. This problem in $\mathbb{R}^{2}$ has a close relation with the explicit expression for the first return map of the differential equation (1.1) (see [10, 12]).

The expression of the first return map can be given in terms of the following iterated integrals of order $k$

$$
I_{i_{1} \cdots i_{k}}(a):=\underbrace{\int \cdots \int}_{0 \leq \tau_{1} \leq \cdots \leq \tau_{k} \leq 2 \pi} a_{i_{k}}\left(\tau_{k}\right) \cdots a_{i_{1}}\left(\tau_{1}\right) d \tau_{k} \cdots d \tau_{1},
$$

where by convention when $k=0$ we set it equal to 1 . By the Ree formula [14] the linear space generated by all such functions is an algebra which is commutative, associative and regularly graded (see [5, p.150] for a definition). More precisely, let $\rho\left(\theta ; \rho_{0} ; a\right)$ with $\theta \in[0,2 \pi]$ and $a(\theta)=\left(a_{1}(\theta), \ldots\right)$ be the solution of equation (1.1) so that $\rho\left(0 ; \rho_{0} ; a\right)=\rho_{0}$. Then the first return map is $P(a)\left(\rho_{0}\right)=\rho\left(2 \pi ; \rho_{0} ; a\right)$ and in $[10,12]$ it is proved that for a sufficiently small $\rho_{0}$ the first return map $P(a)\left(\rho_{0}\right)$ is an absolute convergent power series given by

$$
\rho_{0}+\sum_{n=1}^{\infty} c_{n}(a) \rho_{0}^{n+1}
$$

where

$$
c_{n}(a)=\sum_{i_{1}+\cdots+i_{n}=n} c_{i_{1} \cdots i_{k}} I_{i_{1} \cdots i_{k}}(a),
$$

and

$$
c_{i_{1} \cdots i_{k}}=\left(n-i_{1}+1\right)\left(n-i_{1}-i_{2}+1\right)\left(n-i_{1}-i_{2}-i_{3}+1\right) \cdots 1 .
$$

Received: 17.06.2020. In revised form: 20.01.2021. Accepted: 27.01.2021

2010 Mathematics Subject Classification. 34A05, 34C05.

Key words and phrases. center problem, universal center, complex variables, composition conditions. 
Following the definitions in $\mathbb{R}$ (see [11]) we say that the differential equation (1.1) has a universal center if for all positive integers $i_{1}, \ldots, i_{k}$ with $k \geq 1$ the iterated integral $I_{i_{1} \cdots i_{k}}(a)=0$ (note that in the trigonometric polynomial case, this set needs not be finite).

On the other hand we say that equation (1.1) satisfies the composition conditions if there is a trigonometric polynomial with complex coefficients $q$ and there are polynomials $p_{i} \in$ $\mathbb{C}[w]$, for $i \geq 1$ such that

$$
\tilde{a}_{i}=p_{i} \circ q, \quad \text { where } \quad \tilde{a}_{i}(\theta)=\int_{0}^{\theta} a_{i}(\tau) d \tau, \quad i \geq 1 .
$$

There are plenty of results in the case of $\mathbb{R}$ of results regarding universal centers and composition conditions either for polynomials or analytic functions (see for instance [1, 2, $3,4,6,7,8,9]$ and the references therein), but there are almost no results in the case of $\mathbb{C}$. Brudnyi in [12, Corollary 1.19] proved that equation (1.1) with finitely many $a_{i}{ }^{\prime}$ s being all trigonometric polynomials has a universal center if and only if it satisfies the composition condition (1.3) for all $a_{i}$ with $i=1,2, \ldots, n$. In the present paper we generalize this result to the differential equation (1.1), that is, with infinitely many $a_{i}$ 's (see Theorem 1.1 below). So we extend to an analytic differential equation in $\rho$ as in (1.1). This is done in this paper for the first time

Theorem 1.1. Every center of (1.1) is universal if and only if (1.1) satisfies the composition condition.

The proof of Theorem 1.1 is given in Section 3. In the case of $\mathbb{R}$ this theorem was proved in [13].

In Section 2 we introduce some notation and auxiliary results that will be used in the proof of Theorem 1.1 and whose proofs are given in the Appendix.

\section{Auxiliary Results}

We introduce some notation and auxiliary results that will be used in the proof of Theorem 1.1. Given a trigonometric polynomial $p$ we call $\operatorname{deg}(p)=\ell$ the degree of the Fourier series corresponding to $p$, that is

$$
f(\theta)=\sum_{k=-\ell}^{\ell} a_{k} e^{k i \theta}, \quad a_{k} \in \mathbb{C}, \quad \text { with } a_{\ell}, a_{-\ell} \neq 0 .
$$

To introduce a variant of the Lüroth theorem we introduce some notation. We denote by $\mathbb{C}(x)$ the quotient field of the ring of polynomials $\mathbb{C}[x]$ with coefficients in $\mathbb{C}$ and by $\mathbb{C}(\theta)$ the quotient field of the ring of trigonometric polynomials $\mathbb{C}[\theta]$, also with coefficients in $\mathbb{C}$. It is well-known that $\mathbb{C}(\theta)$ is isomorphic to $\mathbb{C}(x)$ by means of the map $\Phi: \mathbb{C}(\theta) \rightarrow \mathbb{C}(x)$ defined by

$$
\Phi(\sin \theta)=\frac{x^{2}-1}{2 i x} \quad \Phi(\cos \theta)=\frac{x^{2}+1}{2 x} .
$$

Moreover, if we consider the function field $F=\mathbb{C}(x, y)$ with $x^{2}+y^{2}=1$ and the the ring of trigonometric polynomials with complex coefficients $T=\mathbb{C}[x, y]$ (again with $x^{2}+y^{2}=1$ ), then the algebraic curve over $\mathbb{C}$ given by the equation $x^{2}+y^{2}=1$ has no singularities, and so the ring $T$ is integrally closed. Due to the equation

$$
(x+i y)(x-i y)=1 \quad\left(\text { with } i^{2}=-1\right),
$$

if we set $z=x+i y$ then $F=\mathbb{C}(z)$ and $T=\mathbb{C}\left[z, z^{-1}\right]$. In particular, every $t \in T \backslash \mathbb{C}[z]$ has the form $f / z^{m}$, for some $m \in \mathbb{N}$ and $f \in \mathbb{C}[z]$ not divisible by $z$. The first of our auxiliary results is the following. 
We first recall Lüroth's theorem which states that every intermediate field $E$ with $\mathbb{C} \subseteq E \subseteq \mathbb{C}(\theta)$ is a simple transcendental extension, that is $E=\mathbb{C}(s(\theta))$ where $s(\theta)$ is a nonconstant quotient of trigonometric polynomials with coefficients in $\mathbb{C}$. For a proof of Lüroth's theorem see [16, page 21]. We will adapt Lüroth's theorem to our purposes and prove the more convenient following theorem.

Theorem 2.2. An intermediate field $E$ with $\mathbb{C} \subseteq E \subseteq \mathbb{C}(\theta)$ satisfies that $E=\mathbb{C}(r)$ for some non-constant trigonometric polynomial $r$.

Let

$$
\tilde{a}(\theta)=\int_{0}^{\theta} a(s) d s
$$

Lemma 2.1. If equation (1.1) has a universal center, then $\tilde{a}_{i}(\theta)$ is a trigonometric polynomial for all $i \geq 1$.

Given a $k$-vector of indexes $i_{1} i_{2} \cdots i_{k}$ we define

$$
I_{i_{1} i_{2} \cdots i_{k}}(\theta)=\underbrace{\int \cdots \int}_{0 \leq \tau_{1} \leq \cdots \leq \tau_{k} \leq 2 \pi} a_{i_{k}}\left(\tau_{k}\right) \cdots a_{i_{1}}\left(\tau_{1}\right) d_{\tau_{k}} \cdots d_{\tau_{1}}
$$

and if we denote by $\vec{i}=i_{1} i_{2} \cdots i_{k}$ then by (2.4) we have

$$
I_{\vec{i} j}(\theta)=\int_{0}^{\theta} I_{\vec{i}}(\tau) a_{j}(\tau) d \tau
$$

where by convention we have $I_{\emptyset}(\theta)=1$. The Ree's formula (see [14]) establishes a way to write the product of two iterated integrals $I_{\vec{i}}(\theta)$ and $I_{\vec{j}}(\theta)$ as a summation of all the iterated integrals indexed by the shuffle products of the indexes $\vec{i}$ and $\vec{j}$ (we recall that a $(r, s)$-shuffle is a permutation $\sigma$ of $r+s$ letters with $\sigma^{-1}(1)<\sigma^{-1}(2)<\sigma^{-1}(r)$ and $\left.\sigma^{-1}(r+1)<\sigma^{-1}(r+2)<\cdots \sigma^{-1}(r+s)\right)$. More precisely it guarantees that

$$
I_{\vec{i}}(\theta) I_{\vec{j}}(\theta)=\sum_{\sigma} I_{\sigma(\vec{i}, \vec{j})}(\theta),
$$

where the sum runs over all $\sigma(\vec{i}, \vec{j})$ or $(r, s)$-shuffles. For instance, Ree's formula gives that

$$
\begin{aligned}
I_{i_{1}}(\theta) I_{i_{2}}(\theta) & =I_{i_{1} i_{2}}(\theta)+I_{i_{2} i_{1}}(\theta), \\
I_{i}^{m}(\theta) & =m ! \underbrace{I_{\ldots i} \ldots i}_{m \text { times }}(\theta), \\
I_{i_{1}}(\theta) I_{i_{2} i_{3}}(\theta) & =I_{i_{1} i_{2} i_{3}}(\theta)+I_{i_{2} i_{1} i_{3}}(\theta)+I_{i_{2} i_{3} i_{1}}(\theta), \\
I_{i_{1} i_{2}}(\theta) I_{i_{3} i_{4}}(\theta)= & I_{i_{1} i_{2} i_{3} i_{4}}(\theta)+I_{i_{1} i_{3} i_{2} i_{4}}(\theta)+I_{i_{1} i_{3} i_{4} i_{2}}(\theta)+I_{i_{3} i_{1} i_{2} i_{4}}(\theta) \\
& +I_{i_{3} i_{1} i_{4} i_{2}}(\theta)+I_{i_{3} i_{4} i_{1} i_{2}}(\theta),
\end{aligned}
$$

and so on.

As a direct consequence of Ree's formula (2.6) we have the following lemma

Lemma 2.2. There exist non-negative numbers $n_{j}$ for $j=1,2, \ldots, J$ such that

$$
\tilde{a}_{i_{1}}^{m_{1}}(\theta) \tilde{a}_{i_{2}}^{m_{2}}(\theta) \cdots \tilde{a}_{i_{k}}^{m_{k}}(\theta)=\sum_{j=1}^{J} n_{j} I_{\sigma_{j}(\vec{i})}(\theta),
$$

where $i_{j} \geq 1, m_{j} \geq 0$ for $j=1, \ldots, k, \sigma_{j}$ runs over all permutations of the vector

$$
\vec{i}=\underbrace{i_{1} i_{1} \cdots i_{1}}_{m_{1} \text { times }} \underbrace{i_{2} i_{2} \cdots i_{2}}_{m_{2} \text { times }} \cdots \underbrace{i_{k} i_{k} \cdots i_{k}}_{m_{k} \text { times }}
$$


and $J=\left(m_{1}+m_{2}+\cdots+m_{k}\right)$ !

Given equation (1.1) we denote by $\Gamma(a)$ the minimal field containing all the functions $\tilde{a}_{i}(\theta)$ and $\mathbb{C}$. We note that $\Gamma(a)$ is the quotient field of the polynomial domain formed by all the linear combinations with coefficients in $\mathbb{C}$ of monomials of the form

$$
\tilde{a}_{i_{1}}^{m_{1}}(\theta) \tilde{a}_{i_{2}}^{m_{2}}(\theta) \cdots \tilde{a}_{i_{k}}^{m_{k}}(\theta)
$$

where $i_{j} \geq 1$ and $m_{j} \geq 0$ for $j=1,2, \ldots, k$. We consider two polynomials $p(\theta)$ and $q(\theta)$ of $\Gamma(a)$ that is, two functions formed by linear combinations of monomials of the form in (2.8) with coefficients in $\mathbb{C}$.

Lemma 2.3. Consider two polynomials $p_{1}, p_{2} \in \Gamma(a)$. If equation (1.1) has a universal center, then

$$
\int_{0}^{2 \pi} p_{1}(\theta) p_{2}^{\prime}(\theta) d \theta=0
$$

\section{ProOf OF THEOREM 1.1}

Assume first that equation (1.1) satisfies the composition condition

$$
\tilde{a}_{i}(\theta)=p_{i}(q(\theta)), \quad i \geq 1
$$

and we will show that equation (1.1) has a universal center. We take an iterated integral $I_{\vec{i}}(\theta)$ of order $k$ and by induction over $k$ we will show that there exists a polynomial $P_{\vec{i}}(w) \in \mathbb{C}[w]$ such that $I_{\vec{i}}(\theta)=P_{\vec{i}}(q(\theta))$ and $P_{\vec{i}}(q(0)=0$.

When $k=1$, given any index $i \geq 1$ we have

$$
I_{i}(\theta)=\int_{0}^{\theta} a_{i}(\tau) d \tau=\tilde{a}_{i}(\theta)
$$

Thus, since the equation satisfies the composition condition, there exists a polynomial $p_{i}(w) \in \mathbb{C}[w]$ such that $I_{i}(\theta)=p_{i}(q(\theta))$. Note that $p_{i}(q(0))=0$.

Now we assume that the statement holds for $k$ and we will prove it for $k+1$. We take $\vec{i}=i_{1} i_{2} \cdots i_{k}$. By induction hypothesis we have that there exists a polynomial $P_{\vec{i}} \in \mathbb{C}[w]$ such that $I_{\vec{i}}(\theta)=P_{\vec{i}}(q(\theta))$. We consider any index $j \geq 1$ and by (2.5) we have

$$
I_{\vec{i} j}(\theta)=\int_{0}^{\theta} I_{\vec{i}}(\tau) a_{j}(\tau) d \tau
$$

Moreover, since $\tilde{a}_{j}^{\prime}(\theta)=a_{j}(\theta)$ and by the composition condition we know that there exists a polynomial $p_{j}(w) \in \mathbb{C}[w]$ such that $\tilde{a}_{j}(\theta)=p_{j}(q(\theta))$ we get that

$$
I_{\overrightarrow{i j}}(\theta)=\int_{0}^{\theta} P_{\vec{i}}(q(\tau)) p_{j}^{\prime}(q(\tau)) q^{\prime}(\tau) d \tau=P_{\overrightarrow{i j}}(q(\theta))-P_{\vec{i} j}(q(0)),
$$

where $P_{\vec{i} j}(w)$ is a polynomial that is a primitive of the polynomial $P_{\vec{i}}(w) p_{j}^{\prime}(w)$ (that is $P_{\vec{i} j}^{\prime}(w)=P_{\vec{i}}(w) p_{j}^{\prime}(w)$. Without loss of generality we can assume that $P \vec{i} j(w)$ satisfies that $P_{\vec{i} j}(q(0))=0$. We recall that equation (1.1) has a universal center if any iterated integral $I_{\vec{i}}(a)=0$. Given any $\vec{i}$ we have proved that there exists a polynomial $P_{\vec{i}}(w) \in \mathbb{C}[w]$ such that $I_{\vec{i}}(\theta)=P_{\vec{i}}(q(\theta))$ and $P_{\vec{i}}(q(0))=0$. Therefore,

$$
I_{\vec{i}}(a)=I_{\vec{i}}(2 \pi)=P_{\vec{i}}(q(2 \pi)) .
$$

Since $q(\theta)$ is a trigonometric polynomial, we have that $q(2 \pi)=q(0)$ and since $P_{\vec{i}}(q(0))=0$ we obtain that $I_{\vec{i}}(a)=0$. In short, equation (1.1) has a universal center, as we wanted to show. 
Now we prove the converse, that is, we assume that equation (1.1) has a universal center and we will show that it satisfies the composition condition (3.9). If equation (1.1) has a trivial center, that is $\tilde{a}_{i}(\theta) \equiv 0$ for all $i$, then the composition condition trivially holds taking any polynomial $q(\theta)$ and $p_{i} \equiv 0$ for all $i$. We thus assume that equation (1.1) has a nontrivial universal center. As before we denote by $\Gamma(a)$ the minimal field containing all the function $\tilde{a}_{i}(\theta)$ and $\mathbb{C}$.

In order to apply Theorem 2.2 we need to show that $\Gamma(a)$ is an intermediate field between $\mathbb{C}$ and $\mathbb{C}(\theta)$. By definition $\mathbb{C} \subset \Gamma(a)$, but if these two fields were equal then we would have that all the function $\tilde{a}_{i}(\theta)$ would be constant and since $\tilde{a}_{i}^{\prime}(\theta)=a_{i}(\theta)$ we would have a trivial universal center, which we have already discarded. Thus we have that $\mathbb{C} \subsetneq \Gamma(a)$. Moreover, by Lemma 2.1 we have that $\Gamma(a) \subset \mathbb{C}(\theta)$. If these two fields were equal then we would have that the polynomials $p_{1}(\theta)=e^{i \theta}$ and $p_{2}(\theta)=e^{-i \theta}$ belong to $\Gamma(a)$. Then

$$
\int_{0}^{2 \pi} p_{1}(\theta) p_{2}^{\prime}(\theta) d \theta=\int_{0}^{2 \pi}-i d \theta=-2 \pi i,
$$

in contradiction with Lemma 2.3 (this integral should be zero). Therefore, we have $\Gamma(a) \subsetneq$ $\mathbb{C}(\theta)$. It follows from Lüroth's theorem that there exists a nonconstant quotient of trigonometric polynomials $q(\theta)$ such that $\Gamma(a)=\mathbb{C}(q(\theta))$. Since $\Gamma(a)$ contains at least one $\tilde{a}_{i}(\theta)$ which is a non-constant trigonometric polynomial, by Theorem 2.2 we have that there exists a nonconstant trigonometric polynomial $r(\theta)$ such that $\Gamma(a)=\mathbb{C}(r(\theta))$, which proves that equation (1.1) satisfies the composition condition. In short, Theorem 1.1 is proved.

\section{APPENDIX: PROOF OF THE RESUlTS IN SECTION 2}

Proof of Theorem 2.2. We first state and prove two auxiliary results.

Lemma 3.4. A proper subfield $E \neq \mathbb{C}(\theta)$ of $C(\theta)$ is generated by an element $t \in T \backslash \mathbb{C}[z]$ if and only if it is generated by an element of the form

$$
\frac{a f+b z^{m}}{c f+d z^{m}} \quad \text { with } a, b, c, d \in \mathbb{C}, a d-b c \neq 0,
$$

and where $f \in \mathbb{C}[z]$ is not divisible by $z$, and $m \in \mathbb{N}$.

Proof of the lemma. Assume first that $E=\mathbb{C}(t), t=f / z^{m}$ for some $m \in \mathbb{N}, f \in \mathbb{C}[z]$ not divisible by $z$. Every generator of $E$ has the form

$$
\frac{a t+b}{c t+d}=\frac{a \frac{f}{z^{m}}+b}{c \frac{f}{z^{m}}+d}=\frac{a f+b z^{m}}{c f+d z^{m}}
$$

with $a, b, c, d \in \mathbb{C}$ and $a d-b c \neq 0$. For the converse, let $g:=\frac{a f+b z^{m}}{c f+d z^{m}}$ be a generator of $E$. Then

$$
\frac{d g-b}{a-c g}=\frac{f}{z^{m}} \in T
$$

is a generator of $E$. This concludes the proof of the lemma.

Lemma 3.5. A proper subfield $E \neq \mathbb{C}$ of $\mathbb{C}(\theta)$ is generated by an element $t \in T \backslash \mathbb{C}$ if and only if $T \cap E \neq \mathbb{C}$

Proof of the Lemma. Let $t \in T$ be an element of $E \backslash \mathbb{C}$. If $t \in \mathbb{C}[z]$, then [15, Theorem 4, Section 1.2] guarantees that $E$ is generated by a nonconstant polynomial in $z$. If $t \in$ $T \backslash \mathbb{C}[z]$, then let $t=\frac{f}{z^{m}} \in E \backslash \mathbb{C}$ for some $m \in \mathbb{N}$ and $f \in \mathbb{C}[z]$ not divisible by $z$. Let also 
$E=\mathbb{C}\left(\frac{p}{q}\right)$ with coprime polynomials $p, q \in \mathbb{C}[z]$. Then there exist coprime polynomials $G, H \in \mathbb{C}\left[\frac{p}{q}\right]$ such that

$$
\frac{f}{z^{m}}=\frac{G\left(\frac{p}{q}\right)}{H\left(\frac{p}{q}\right)} \text {. }
$$

Since both polynomials $G$ and $H$ split into linear factors of the form $\frac{p}{q}-\gamma$ for some $\gamma \in \mathbb{C}$ and $\frac{p}{q}-\gamma=\frac{p-\gamma q}{q}$ one gets

$$
\frac{f}{z^{m}}=q^{n} \frac{\prod_{i=1}^{r}\left(p-\alpha_{i} q\right)^{c_{i}}}{\prod_{j=1}^{s}\left(p-\beta_{j} q\right)^{d_{j}}},
$$

for some $n \in \mathbb{Z}, r, s, c_{i}, d_{j} \in \mathbb{N}$ and where $\alpha_{i}, \beta_{j}$ are pairwise distinct.

Note that by assumption the polynomials $p-\alpha_{i} q$ and $p-\beta_{j} q$ have no zeros in common with $q$ and that the two polynomials $p-\alpha q$ and $p-\beta q$ with $\alpha \neq \beta$ have no common zeroes. Hence, in equation (3.10) no linear factors can cancel out and since the denominator of the left-hand-side of equation (3.10) has $\mathrm{z}$ as its only linear factor, only the following three cases are possible: either the polynomial $H$ is constant, or $s=1$ (note that if $m=0$ then $H$ must be constant).

In case $H$ is constant then $n<0$ and so $q=z^{\ell}$ for some $\ell \in \mathbb{N}$ and so $\frac{p}{q} \in T$.

In case $s=1$ we have that $p-\beta_{1} q=z^{\ell}$ for some $\ell \in \mathbb{N}$ and therefore $\frac{p}{q}=\frac{\beta_{1} q+z^{\ell}}{q}$. It follows from Lemma 3.4 that $\frac{q}{z^{\ell}} \in T$ is a generator of $\mathrm{E}$. This concludes the proof of the lemma.

The proof of Theorem 2.2 follows directly from Lemma 3.5

Proof of Lemma 2.1. Given an index $i \geq 1$ we consider the expansion in Fourier series of the coefficient $a_{i}(\theta)$ and we denote by $\ell_{i}$ the degree of the trigonometric polynomial $a_{i}(\theta)$. Then

$$
a_{i}(\theta)=\sum_{n=-\ell_{i}}^{\ell_{i}} c_{n i} e^{i n \theta},
$$

where $c_{n i} \in \mathbb{C}$ for all $i \geq 1$. We have that

$$
\tilde{a}_{i}(\theta)=\int_{0}^{\theta} a_{i}(\tau) d \tau=c_{0 i} \theta+\sum_{n=-\ell_{i}, n \neq 0}^{\ell_{i}} \frac{1}{n i} c_{n i}\left(e^{i n \theta}-1\right) .
$$

Since equation (1.1) has a universal center and $\tilde{a}_{i}(2 \pi)=I_{i}(a)$ we have that $\tilde{a}_{i}(2 \pi)=0$ and so $c_{0 i}=0$. Hence $\tilde{a}_{i}(\theta)$ is a trigonometric polynomial.

Proof of Lemma 2.3. Take $p_{1}(\theta)$ a monomial of the form (2.8) and $p_{2}(\theta)=\tilde{a}_{k}(\theta)$ for some index $k \geq 1$. Then

$$
\int_{0}^{\theta} p_{1}(\tau) p_{2}^{\prime}(\tau) d \tau=\int_{0}^{\theta} \tilde{a}_{i_{1}}^{m_{1}}(\tau) \tilde{a}_{i_{2}}^{m_{2}}(\tau) \cdots \tilde{a}_{i_{k}}^{m_{k}}(\tau) a_{k}(\tau) d \tau .
$$

By Lemma 2.2 we have that (2.7) holds and so

$$
\int_{0}^{\theta} p_{1}(\tau) p_{2}^{\prime}(\tau) d \tau=\sum_{j=1}^{J} n_{j} \int_{0}^{\theta} I_{\sigma_{j}(\vec{i})}(\tau) a_{k}(\tau) d \tau .
$$

Moreover, by the relation (2.5) we have

$$
\int_{0}^{\theta} I_{\sigma_{j}(\vec{i})}(\tau) a_{k}(\tau) d \tau=I_{\sigma_{j}(\vec{i}) k}(\theta)
$$


Since equation (1.1) has a universal center we have that $I_{\sigma_{j}(\vec{i}) k}(2 \pi)=0$ and so

$$
\int_{0}^{2 \pi} p_{1}(\tau) p_{2}^{\prime}(\tau) d \tau=\sum_{j=1}^{J} n_{j} \cdot 0=0
$$

as we wanted to show.

\section{Acknowledgements.}

The author is grateful to the reviewers which helped her to improve the paper. The author is partially supported by FCT/Portugal through UID/MAT/04459/2019.

\section{REFERENCES}

[1] Alwash, M. A. M., On a condition for a centre of cubic nonautonomous equations, Proc. Roy. Soc. Edinburgh Sect. A., 113 (1989), 289-291

[2] Alwash, M. A. M., On the composition conjectures, Electron. J. Differential Equations, 69 (2003), 4 pp. (electronic)

[3] Alwash, M. A. M., The composition conjecture for Abel equation, Expo. Math., 27 (2009), 241-250

[4] Alwash, M. A. M. and Lloyd, N. G., Nonautonomous equations related to polynomial two-dimensional systems, Proc. Roy. Soc. Edinburgh Sect. A., 105 (1987), 129-152

[5] Chevalley, C., Fundamental concepts of algebra, Academic Press Inc., New York, 1956

[6] Briskin, M., Françoise, J. P. and Yomdin, Y., Center conditions, compositions of polynomials and moments on algebraic curves, Ergodic Theory \& Dynam. Systems, 19 (1999), 1201-1220

[7] Briskin, M., Françoise, J. P. and Yomdin, Y., Center conditions. II. Parametric and model center problems, Israel J. Math., 118 (2000), 61-82

[8] Briskin, M., Françoise, J. P. and Yomdin, Y., Center conditions. III. Parametric and model center problems, Israel J. Math., 118 (2000), 83-108

[9] Briskin, M., Roytvarf, N. and Yomdin, Y., Center conditions at infinity for Abel differential equations, Ann. of Math., 172 (2010), 437-483

[10] Brudnyi, A., An explicit expression for the first return map in the center problem, J. Differential Equations 206 (2004), 306-314

[11] Brudnyi, A., An algebraic model for the center problem, Bull. Sci. Math., 128 (2004), 839-857

[12] Brudnyi, A., On the center problem for ordinary differential equations, Amer. J. Math., 128 (2006), 419-451

[13] Giné, M., Grau, M. and Llibre, J., Universal centers and composition conditions, Proc. Lond. Math. Soc., 106 (2013), 481-507

[14] Ree, R., Lie elements and an algebra associated with shuffles, Ann. of Math., 68 (1958), 210-220

[15] Schinzel, A., Polynomials with Special Regard to Reducibility, Encyclopedia of Mathematics and its Applications, 77, Cambridge University Press, Cambridge, 2000

[16] van Der Waerden, B. L., Algebra. Vol. I. Based in part of lectures by E. Artin and E. Noether. Translated from the seventh German edition by Fred Blum and John R. Schulenberger, Springer-Verlag, New York, 1991. ISBN:0-387-40624-7

\section{UNIVERSIDADE DE LISBOA}

Departamento de MATEMÁtica, InSTITUTO SUPERIOR TÉCNico

Av. Rovisco PAIS 1049-001, LisboA, PORTUGAL

Email address: cvalls@math.tecnico.ulisboa.pt 